\title{
Kernos
}

Revue internationale et pluridisciplinaire de religion grecque antique

$26 \mid 2013$

Varia

\section{Aspects de la divination dans la monarchie macédonienne}

\section{Elias Koulakiotis}

\section{OpenEdition \\ Journals}

Édition électronique

URL : http://journals.openedition.org/kernos/2206

DOI : 10.4000/kernos.2206

ISSN : 2034-7871

\section{Éditeur}

Centre international d'étude de la religion grecque antique

\section{Édition imprimée}

Date de publication : 10 octobre 2013

Pagination : 123-138

ISSN : 0776-3824

\section{Référence électronique}

Elias Koulakiotis, «Aspects de la divination dans la monarchie macédonienne », Kernos [En ligne], 26। 2013, mis en ligne le 31 octobre 2015, consulté le 02 mars 2021. URL : http://

journals.openedition.org/kernos/2206 ; DOI : https://doi.org/10.4000/kernos.2206 


\title{
Aspects de la divination dans la monarchie macédonienne
}

Résumé: L'article examine les dimensions politiques et religieuses de l'image d'Alexandre le Grand qui, à un moment donné de l'expédition asiatique, interprète lui-même un signe. Il semble que nous avons affaire à un roi-exégète, situé dans le contexte d'une monarchie dont le souverain était aussi le magistrat religieux suprême. Il s'agit d'une image qui invite à réfléchir sur l'importance de la divination à la cour macédonienne, sur les personnes qui y sont impliquées, ainsi que sur les rapports entre pratiques divinatoires grecques, babyloniennes et romaines.

\begin{abstract}
This article examines the political and religious dimensions of the image of Alexander the Great who, at a certain point in his Asian campaign, interprets a sign on his own. It seems that we are dealing with a king who is placed in the context of a monarchy whose head is also the head of religious affairs. This is an image that makes us think about the importance of divination in the Macedonian court, about the persons involved, as well as about the links between the religious practices of Greece, Babylon and Rome.
\end{abstract}

\section{Le royaume et la divination}

Selon les sources littéraires, l'existence du royaume macédonien est dès le début liée à l'action divinatoire. Dans toutes les versions de la fondation du royaume, un signe ou un oracle envoyé par les dieux doit être interprété de la bonne manière par des descendants d'Héraclès pour que le territoire macédonien soit défini ou colonisé ${ }^{1}$. D'après le témoignage d'Hérodote, qui au milieu du ve siècle av. J.-C. rapporte la plus ancienne version du mythe de la famille royale macédonienne ${ }^{2}$, l'acte constitutif du royaume aurait été lié à une série de prodiges

\footnotetext{
${ }^{1}$ La plupart des textes présentés ici concernent la dynastie des Téménides, appelés également à partir du IVe s. av. J.-C. Argéades. Sur les oracles de Delphes concernant la Macédoine, voir PARKe - Wormell (1956), nos 225-227; Fontenrose (1978), L 50-51. Voir aussi KAPETANOpoulos (2010), qui donne une liste de tous les témoignages et MALLIOS (2011), p. 193-195, qui compare toutes les versions.

${ }^{2}$ Selon l'ordre chronologique d'apparition dans les sources, cette première version reconnait Perdiccas comme fondateur de la dynastie et destinataire privilégié d'un signe divin; elle est rapportée par Hérodote, VIII, 138-139 et Thucydide, II, 99, et est datée par Solin, 9, 13 à la secunda et vicesima olympiade. L'oracle relatif à la fondation d'Aigai est absent de cette version dans les récits du ve siècle, et il n'est mentionné que par Diodore, VII, 16. Comme la remarque en a déjà été faite, la version d'Hérodote ne mentionne pas l'oracle de Delphes reçu par Perdiccas; il est possible que l'oracle rapporté par Diodore ait fait partie du mythe original, mais cela ne peut être prouvé (voir MARI [2002], p. 21, qui insiste sur la relation d'Hérodote avec Alexandre Ier de Macédoine). À mon avis, il faudrait plutôt y voir une réélaboration tardive, afin de souligner les
} 
concernant le futur premier roi du pays, l'Argien Perdiccas, descendant de Téménos, et par lui d'Héraclès ${ }^{3}$ : Perdiccas et ses deux frères, Aéropos et Gauanes, s'étaient enfuis d'Argos ${ }^{4}$ et étaient passés au service d'un roi local de Macédoine, qui reste anonyme; celui-ci avait fini par les maltraiter et les chasser, parce que sa femme » lorsqu'elle faisait le pain, la miche destinée au garçon, leur domestique, doublait régulièrement de volume; comme c'était chaque fois la même chose, la reine en informa son mari. Averti, le roi eut aussitôt l'idée qu'il y avait là quelque prodige et l'annonce de choses graves $»^{5}$. Le prodige (teras) se réalise un peu plus tard dans la narration d'Hérodote, quand le roi, frappé et égaré par un dieu (théoblabès) ${ }^{6}$, n'arrive pas à comprendre un geste symbolique du jeune Perdiccas, quand ce dernier « de son couteau traça sur le sol les contours de la tache de soleil; après quoi il fit à trois reprises le geste de puiser du soleil et de verser dans le pli de sa tunique, puis il s'en alla, et ses frères avec lui ».7

Ce geste est interprété (semainei) par un conseiller du roi d'une façon qui irrite le roi, sans doute parce qu'il se sent menacé ${ }^{8}$. En effet, il va bientôt perdre son trône et céder son pouvoir à ses anciens serviteurs. Ainsi, par une action

liens des Téménides avec Delphes. Sur Hérodote et les oracles en général, voir HaRRISON (2000), p. 122-157. Selon la plupart des chercheurs, la première version doit être la plus ancienne et la plus fiable : voir SPRAWSKI (2010), p. 130; voir aussi HAMMOND - GRIFFITH (1979), p. 3-10; Greenwalt (1986); Reynolds (1990), p. 6; Chevutschi (1991), p. 63-70; Tripodi (1993), p. 1624-1625; GREENWALT (1994); MARI (2002), p. 19-22; HatZopoulos (2003). Sur le contexte historique voir aussi PABST (2009). Pour une analyse littéraire du mythe en rapport avec les contes populaires voir KAZAZIS (1978), p. 101-104. Pour une analyse structurale du mythe: TRIPODI (1993) et THEML (2000) et en général SourvinOu-INWOOD (2002)

${ }^{3}$ Hdt, VIII, 137-138.

${ }^{4}$ Sur la localisation d'Argos voir Hatzopoulos (2003), p. 216-218, qui accepte l'identification avec Argos Orestikon.

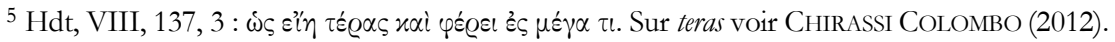

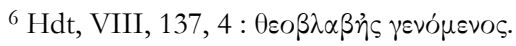

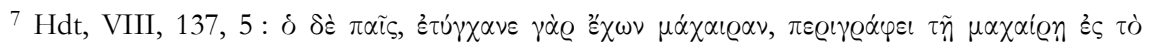

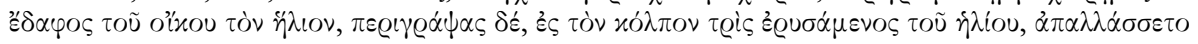

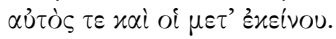

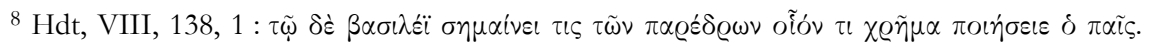
Voir Rhomaios (1981), p. 264, 275 et CheVuschi (1991), p. 64-65 qui soulignent l'aspect prophétique du geste de Perdiccas, ainsi que KLEINKNEChT (1966) qui renvoie à un contexte iranien et magique; voir le parallèle rapporté par Cicéron, De div. I, 24 : Quid ego, quae magi Cyro illi principi interpretati sint, ex Dinonis Persicis fibris proferam? Nam cum dormienti ei sol ad pedes visus esset, ter eum scribit frustra adpetivisse manibus, cum se convolvens sol elaberetur et abiret; ei magos dixisse, quod genus sapientium et doctorum babebatur in Persis, ex triplici adpetitione solis triginta annos Cyrum regnaturum esse portendi. Ici, le geste de Cyrus est comparable à celui de Perdiccas et prédit l'avenir du roifondateur de la monarchie perse; voir FrEYBURGER - SCHEID (1992), p. 49, n. 101 et 102. Sur le culte du soleil en Macédoine, voir BAEGE (1913), p. 128-130 et DüLL (1977), p. 134. Sur la présence du soleil dans le mythe fondateur et l'emblème de la dynastie royale macédonienne, voir TRIPODI (1986); RitTER (1981); voir aussi LIAMPI (1998), p. 43-44 sur le lien douteux entre la

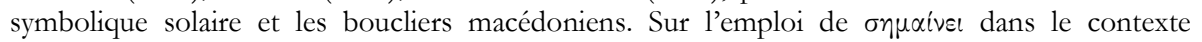
oraculaire voir aussi GEORGOUDI (2012), p. 64-68. 
qui possède certains traits magiques et propres à une divination 'déductive', la prise de pouvoir de Perdiccas et ses frères sur le pays de leur ancien maître est légitimée.

Ensuite, vers la fin du $\mathrm{V}^{\mathrm{e}} \mathrm{s}$. Euripide semble avoir réélaboré le mythe des Téménides et présenté un autre fils de Téménos, Archélaos, en tête de la dynastie ${ }^{10}$; celui aurait reçu un oracle pour fonder Aigai, la capitale du royaume.

En dernier lieu, d'après les témoignages postérieurs à Hérodote et à Euripide, rapportés surtout par Théopompe et Justin (qui cite Marsyas de Pella, auteur de la fin du IV e s. av. J.-C.), c'est de nouveau un descendant de Téménos, non pas Perdiccas, mais son père Caranos, qui aurait reçu un oracle lui enjoignant de s'installer au nord de l'Olympe ${ }^{11}$ : «Enfin Caranus, sur une réponse de l'oracle qui lui ordonnait de chercher un établissement en Macédoine, vint en Émathie à la tête d'une nombreuse colonie grecque $»^{12}$.

Tous ces textes, issus dans des moments différents du discours de légitimation de la royauté macédonienne ${ }^{13}$, témoignent de l'importance de la divination dans ce discours et ils véhiculent l'image d'un roi-fondateur qui a des relations privilégiées au monde divin. Or, cette image ne peut qu'avoir un rôle normatif pour les successeurs de Perdiccas, Archélaos et Caranos. Il faudrait éventuellement voir dans ces trois protagonistes des aspects complémentaires d'un même personnage ${ }^{14}$, c'est-à-dire un roi héroïque, fondateur de la monarchie macédonienne: Perdiccas et ses avatars, Archélaos et surtout Caranos, incarnent le

9 La qualification est selon la classification de Cicéron: voir CHIRASSI CoLOMBO (2012), p. 221-225. Sur la problématique de la catégorisation des modes divinatoires voir la discussion chez GEORGOUdi (2003), p. 10-12 et BuRKERT (2005), p. 2-3.

${ }^{10}$ Cette seconde version place Archélaos, fils de Téménos, à la tête de la dynastie et fut très probablement fabriquée par Euripide vers la fin du ve s. av. J.-C., pendant son séjour à la cour du roi macédonien Archélaos: voir Hygin, fab. 219, qui sans doute donne le résumé d'Archélaos, tragédie perdue d'Euripide. L'homonymie d'Archélaos qui régnait à l'époque en Macédoine est évidemment révélatrice. Sur Archélaos voir XANTHAKI-KARAMANOU (1993) et COLLARD - CROPP - Gibert (2005), p. 330-332. Sur Euripide et ses liens avec la maison royale de Macédoine, voir HARDER (1991) et SCULLION (2003), p. 389-391, qui n'admet pas l'historicité du séjour d'Euripide en Macédoine. Il semble que Dion Chrys., IV, 70-72 s’inspire de cette deuxième version.

${ }^{11}$ Cette troisième version attribue à Caranos le rôle de père de Perdiccas et de fondateur d'un centre urbain à Aigai; elle est rapportée par Plut., Alex. II, 1, mais connue déjà par Théopompe, FGrHist 115 F 93 et Marsyas, FGrHist 135-136 F 14 (cf. Justin, VII 1, 7).

12 Justin, VII, 1, 7-12.

13 Sur les dimensions politiques du mythe fondateur de la dynastie téménide, voir ASIRVATHAM (2010), p. 100-102 et MALLIOS (2011), p. 175-229. Sur l’importance des récits généalogiques pour la construction d'une identité collective, voir MülLER (2009) et ENGELS (2010). Voir aussi BRANDENSTEIN (1954), ZAHRNT (1984) et OGDEN, (2011), p. 57-78.

14 TRIPODI (1993), p. 1627-1630 évoque une «typologie » du roi-fondateur qui concerne tous les membres du génos des Téménides. D’après Tripodi, la présence de machaira dans le récit d'Hérodote (VIII, 137, 5), renvoie à l'action sacrificielle. À part cela, le caractère magico-sacral de Perdiccas dans le chapitre 137 du récit hérodotéen souligne son identité de roi-prêtre, tandis que dans le chapitre 138, c'est l'aspect militaire et conquérant qui est mis en valeur. Voir aussi THEML (2000), p. 52. 
héros qui à la fois reçoit des prodiges et des oracles, et sait les interpréter et les réaliser correctement ${ }^{15}$.

Qui plus est, selon la version de Justin, Perdiccas, vers la fin de sa vie et juste avant de mourir, semble exercer lui-même une fonction oraculaire, lorsqu'il prédit la fin de sa propre dynastie au cas où les corps des rois morts ne seraient pas enterrés à Aigai, première capitale et "ville sacrée » du royaume ${ }^{16}$ : "Après lui régna Perdiccas, dont la vie fut illustre et dont les dernières recommandations s'imposèrent à la mémoire, comme si elles venaient d'un oracle ${ }^{17}$. Cette "prophétie ", de la bouche de quelqu'un qui va bientôt appartenir au monde des morts, n'est pas un cas unique, et il est certain que l'épisode fut composé après la mort d'Alexandre. Néanmoins, elle participe de l'image des rois macédoniens que l'on vient d'esquisser, et il faudrait l'inscrire dans cette tradition.

Tous ces témoignages indiquent une relation privilégiée entre les rois macédoniens et les dieux, et renvoient aux traits caractéristiques de cette sorte de royauté qu'Aristote appelle « héroïque » : «Dans une quatrième sorte de monarchie royale on trouve celle des temps hérö̈ques établie sous le contrôle de la loi, à la fois consentie et héréditaire. Car du fait que les premiers membres du lignage avaient rendu service au peuple dans le domaine des arts ou de la guerre, ou avaient rassemblé le peuple ou lui avaient fourni un territoire, ils devinrent rois de gens consentants, et cette fonction devint héréditaire pour ceux qui leur succédèrent. Ils étaient maitres de la conduite de la guerre, de l'organisation de ceux des sacrifices qui ne relevaient pas de prêtres, et, outre cela, ils jugeaient les procès $»^{18}$. Le philosophe résume plus loin : «le roi était stratège, juge et maitre du culte des dieux ».

Dans la présente étude, je propose justement d'examiner la relation entre une royauté qui se voulait hérö̈que ${ }^{19}$ et les pratiques liées au culte des dieux et à la divination, notamment le sacrifice et l'interprétation de signes. C'est surtout à travers l'exemple d'Alexandre le Grand que j'aimerais tracer quelques jalons de l'évolution de cette image.

15 À confronter au cas d'Hyllos, fils d'Héraclès, qui n'a pas su interpréter un oracle et a conduit l'armée au désastre : MARI (2002), p. 22. Voir aussi SOURVINOU-INWOOD (2005).

${ }^{16}$ Cf. le témoignage de Diod., XXII, 12. Sur Vergina/Aigai, voir dernièrement Drougou (2011); GALANAKIS (2011) et KOTTARIDI (2011 b).

${ }^{17}$ Justin, VII, $1,8$.

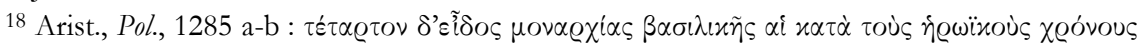

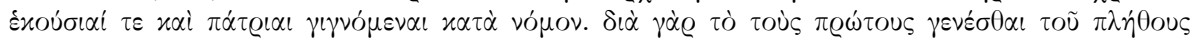

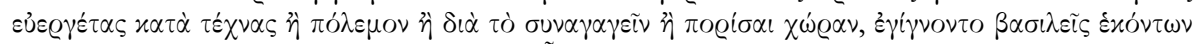

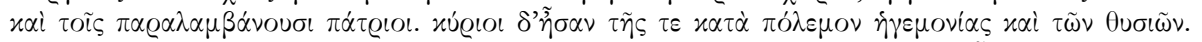

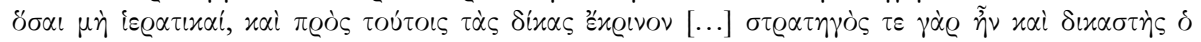

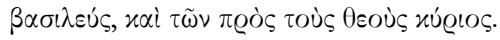

${ }^{19}$ Sur cet aspect du caractère de la royauté macédonienne, voir CARLIER (2000); KING (2004), p. 41 et KING (2010), p. 375-376. 


\section{Le maître du culte des dieux et les pratiques divinatoires}

Le point de départ de cette étude est l'un des derniers épisodes de la vie d'Alexandre, qui survint peu de temps avant sa mort. Le contexte historique est celui du retour du roi à Babylone, en 323 av. J.-C., et de son entrée dans la ville ${ }^{20}$. Ce retour, après l'expédition en Inde, constituait pour plusieurs stratèges et satrapes une mauvaise nouvelle. La présence du conquérant signifiait qu'ils risquaient de devoir rendre compte de leur gestion, de subir un contrôle concernant leur administration, et éventuellement d'être sévèrement punis, s'ils n'avaient pas observé les prescriptions du roi. L'incident est rapporté par Plutarque et par Arrien ${ }^{21}$, il fait partie des omina mortis d'Alexandre ${ }^{22}$, et il vaut la peine de comparer les récits de ces deux auteurs ${ }^{23}$.

Selon Arrien ${ }^{24}$, dont le texte est postérieur à celui de Plutarque, mais qui utilise comme source Aristobule ${ }^{25}$, historien compagnon d'Alexandre dont le texte est perdu :

Apollodore d'Amphipolis, un des Compagnons d'Alexandre, qui commandait l'armée qu'Alexandre avait laissée derrière lui auprès de Mazéos, satrape de Babylone, se trouvant avec Alexandre à son retour de l'Inde, et voyant qu'il châtiait sévèrement les satrapes qu'il avait mis à la tête des différentes provinces, écrivit à son frère Peithagoras, devin qui lisait l'avenir dans les entrailles des victimes, pour lui demander une prédiction concernant son propre salut. Peithagoras lui repondit en lui demandant de qui il avait particulièrement peur pour recourir ainsi à une consultation. Apollodore lui écrivit de nouveau en lui disant que c'était du roi luimême et d'Héphestion.

À la suite de cet échange, Peithagoras rassura son frère : il prédisait à la fois la mort d'Héphéstion et celle d'Alexandre, puisque pour tous deux les foies des victimes immolées n'avaient pas de lobe. Arrien poursuit :

\footnotetext{
${ }^{20}$ Sur le contexte voir BOSWORTH (1988), p. 158-173, ainsi que BRIANT (1999).

${ }^{21}$ L'épisode n'est pas mentioné par la 'Vulgate' d'Alexandre (Diodore, Quinte Curce, Justin), mais se trouve chez Appien, $B C$ II, 152, qui reprend les informations d'Arrien; voir WIRTH (1964), p. 209-210 et BOSWORTH (1980), p. 9.

22 Voir SMELIK (1978/79) et ZAMBrini (2007), p. 218-219.

${ }^{23}$ L'incident a été analysé selon un autre point de vue par ABRAMENKo (2000), qui l'inscrit dans le cadre d'un complot contre Alexandre. À mon avis cette interprétation ne peut pas être entièrement acceptée et j'aurai l'occasion de présenter ailleurs mes arguments.

24 Arrien, Anab., VII, 18, 1.

${ }^{25}$ Aristobule, qui en général présente Alexandre comme un roi modéré, semble être le seul qui avait racconté cet épisode. Le point de vue d'Aristobule fut adopté par Arrien, mais pas par Plutarque. Ce dernier, même s'il se réfère à Aristobule dans cette partie de son œuvre (voir Plut., Alex. 75, 6), semble avoir d'autres sources aussi. Voir HAMilton 1999, p. lx-lxii, Auberger (2001), p. 429, n. 415, ainsi que PEDECH (1984), p. 376-377. Sur les sources d'Arrien voir dernièrement CARNeY (2010). Sur Plutarque historien, voir en général PELLING (2002).
} 
Mais Apollodore ne garda pas la nouvelle pour lui; il la communiqua à Alexandre, dans l'idée de montrer au roi son empressement, en lui recommandant de prendre garde de ne pas s'exposer au danger en ce moment. (...) Alexandre félicita Apollodore et, quand il fut arrivé à Babylone, il demanda à Peithagoras quel présage il avait observé pour avoir écrit cela à son frère; et l'autre lui repondit qu'il s'était trouvé devant un foie sans lobe. Comme Alexandre lui demandait ce que signifiait ce présage, Peithagoras répondit: "Quelque chose de très grave.»Et Alexandre, loin de lui en tenir rigueur, lui montra une bienveillance particulière pour lui avoir dit franchement la vérité. Cela, Aristobule déclare l'avoir appris lui-même de la bouche de Peithagoras; il ajoute qu'il fit aussi des prédictions plus tard pour Perdiccas et Antigone; il trouva ce même présage pour tous les deux, et Perdiccas trouva la mort au cours d'une campagne contre Ptolémée [en 321 av. J.-C.], et Antigone dans le combat qui l'opposait à Séleucos et à Lysimaque, et qui a eu lieu à Ipsos [en 301 av. J.-C.].

Le récit de cet épisode par Plutarque ${ }^{26}$ est moins détaillé, mais à mon avis plus intéressant. Nous lisons dans la Vie d'Alexandre:

Quand le roi fut rentré à Babylone, il reçut une dénonciation contre Apollodore, stratège de Babylone, qui avait fait un sacrifice pour connaittre l'avenir à son sujet, et il fit appeler le devin Pythagoras [ou Peithagoras] d'Amphipolis. Celui-ci ne nia point le fait, et, Alexandre le questionnant sur l'état des victimes, il répondit que leur foie était sans lobe : «Grands dieux, s'écria Alexandre, ce présage est grave ! »Il ne fit aucun mal à Pythagoras, mais il regretta de n’avoir pas écouté Néarque, et il passa presque tout son temps à camper hors de Babylone ou à naviguer sur l'Euphrate.

Le texte de Plutarque révèle l'image d'un roi, qui, dans la tradition des maittres de vérité ${ }^{7}$ et conforme à la royauté héroïque d'Aristote, interprète lui-même les signes $^{28}$. Ici se pose la question de savoir lequel de ces deux récits, celui d'Arrien ou celui de Plutarque, est le plus fiable, question qui n'est pas facile à résoudre ${ }^{29}$. Car, nous avons affaire à des narrations qui ont chacune leur propre raison d'être ${ }^{30}$, et même si la signification de certains présages constituait jusqu'à un certain point un topos littéraire ${ }^{31}$, il faut resituer l'image du roi-exégète qu'elles présentent dans le contexte d'une monarchie dont le souverain était aussi le magistrat religieux suprême. Cependant, il faut aussi prendre en considération le fait qu'à cette époque Alexandre n'était pas seulement un roi macédonien, mais aussi un monarque orientalisé. L'« orientalisation » d'Alexandre est un vaste sujet

26 Plut., Alex., 73, 1-4.

27 Voir Detienne (1973).

28 Voir les remarques de KING (2004), p. 123, au sujet du rôle joué par la divination pendant cette période dans la vie d'Alexandre, pendant laquelle il semble frappé par la superstition. Sur la religiosité d'Alexandre voir aussi AUBRIOT (2003).

${ }^{29}$ Voir Pearson (1960), p. 182 et Hamilton (1999), p. lxi et p. 203.

${ }^{30}$ Voir la discussion sur la polarité entre critique des sources et critique littéraire chez FLOWER (2007), p. 423.

31 Sur le foie sans lobe comme topos littéraire : BONNECHERE (2010), p. 152. 
qui va de pair avec sa divinisation : il s'agit d'une évolution sans doute liée à sa visite au sanctuaire de Zeus Ammon à Siwah, mais peut-être commencée bien avant $^{32}$. Dans cette évolution, la mère d'Alexandre, Olympias, joua peut-être un rôle important ${ }^{33}$. Il semble établi qu'elle avait suggéré à Alexandre son identification à Achille ${ }^{34}$ et qu'elle avait fait enseigner au jeune prince une série de rituels religieux propres à la monarchie macédonienne, dont, selon Athénée, les argadistik. $a^{35}$. Le mot est un hapax et on ne sait pas exactement ce qu'il pouvait signifier ${ }^{36}$. À mon avis, il faudrait y voir un rapport avec les Argéades, et plus

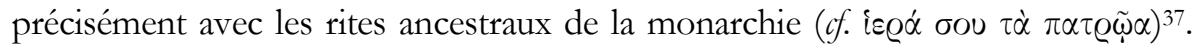
Certes, Olympias venait d'une autre dynastie, mais si on prend en considération le rôle des femmes dans la dynastie macédonienne ${ }^{38}$, l'épisode pourrait s'expliquer : il faut ici songer à Thyia, mère de Makédon, héros éponyme des Macédoniens, dont le nom pourrait faire allusion au service sacrificiel ${ }^{39}$.

Il est bien connu que, plus l'expédition d'Alexandre progressa vers l'est, plus le roi s'éloigna de sa simple « humanité ». Cela se traduisait aussi par l'assimilation de rôles nouveaux, comme celui d'héritier des Achéménides ${ }^{40}$. Il est également connu que les souverains perses revendiquaient, eux aussi, une relation privilégiée au monde divin ${ }^{41}$. D’après Xénophon, les Achéménides cherchèrent à préserver cette relation, et ils étaient conscients du rôle d'intermédiaires quelquefois douteux et dangereux des prêtres et des devins. C'est pourquoi nous lisons dans la Cyropédie de Xénophon que le père de Cyrus le Grand lui avait enseigné comment interpréter les signes venus des dieux et les entrailles des victimes pour qu'il puisse affronter de semblables situations ${ }^{42}:$ «... Je t'ai en effet instruit à

32 Voir à titre indicatif FredrickSMEYer (2003), p. 270-278 et Buraselis - ANEZIRI (2005).

33 CARNeY (2006), p. 88-103. Sur les prodiges concernant la naissance d'Alexandre voir OGDEN (2011), p. 7-56.

34 Sur Alexandre nouvel Achille, voir Koulakiotis (2006), p. 39-43.

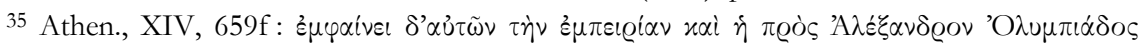

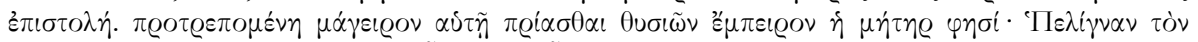

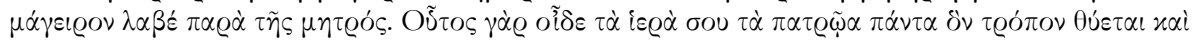

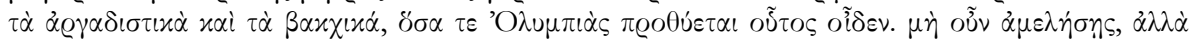

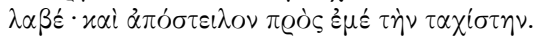

36 Voir FREDRICKSMEYER (1966).

${ }^{37}$ Dans cette perspective, voir aussi KOTTARIDI (2011a), p. 103.

38 CARNeY (2006), p. 95. Sur le rôle des femmes dans la vie religieuse voir aussi LE BOHECBOUHET (2006), p. 190.

${ }^{39}$ Hésiod., fr. 3 Merkelbach-West. Sur Thyia, voir KotTARIDi (2011), p. 1.

40 BRIANT (1996), p. 595-601.

${ }^{41}$ KunRT (2007), p. 548-555.

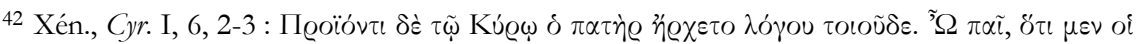

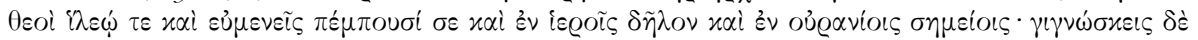

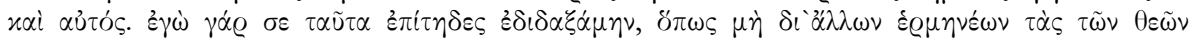

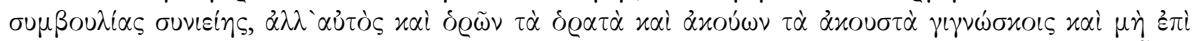

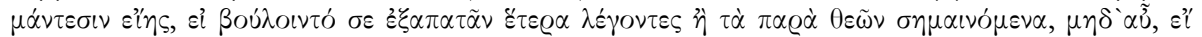

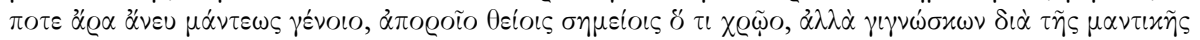


dessein de ces choses, afin que tu n'aies pas besoin d'interprètes pour comprendre les avis des dieux et que tu les reconnaisses par toi-même... ». De cette façon, l'expertise des prêtres et des devins ainsi que leur influence politique se trouvait sous contrôle, et la communication avec les dieux pouvait être réglementée ${ }^{43}$.

Il semble donc qu'il existe dans nos sources une convergence entre les images royales macédonienne et achéménide pour ce qui a trait à la capacité de bien interpréter les signes venus des dieux, image qui a sans doute affaire non seulement avec le rôle religieux et politique des monarques, mais aussi avec leur rôle de chef de l'armée. L'importance de la divination dans le domaine militaire, et sur le champ de bataille même, est connue : le roi-stratège se devait d'avoir une bonne compréhension de la volonté des dieux pour la communiquer aux soldats ${ }^{44}$. De nouveau, le témoignage de Xénophon semble pertinent. Même si cette fois nous n'avons pas affaire au monde de la monarchie mais au monde des cités, l'Anabase des dix mille nous fournit l'image d'un stratège qui, à travers son expérience militaire a acquis une certaine expertise divinatoire ${ }^{45}$. Mis à part le grand problème de l'éducation posé très souvent au IV siècle av. J.-C., il apparaît donc que la capacité (technè) à interpréter les présages et les entrailles pouvait être apprise par les membres d'une dynastie ou par certains citoyens d'une cité. Par conséquent, la version par Plutarque de l'épisode mentionné plus haut pourrait posséder un fond de vérité : Alexandre, en sa capacité de monarque et de stratège, pouvait faire l'interprétation des victimes des sacrifices.

\section{Entre cité et cour : la consultation de Pythagore}

Quant au devin Pythagore (Peithagore ou Peithagoras), qui semble avoir joué un rôle important dans l'histoire des Diadoques ${ }^{46}$, son origine, sa carrière et également son nom méritent d'être étudiés ${ }^{47}$. Sa carrière l'attira dans des cités aussi bien qu'à la cour royale, selon un parcours personnel qui illustre bien la dualité du royaume de Macédoine, dont les cités et ethnè se mettaient au service du pouvoir central ${ }^{48}$. Étant donné que nous sommes encore loin de disposer d'une

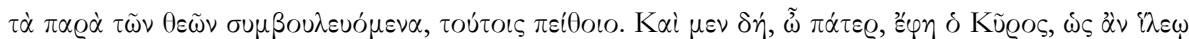

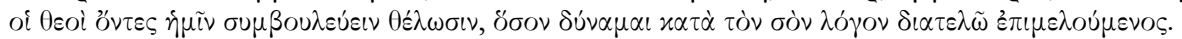

43 Sur ce sujet, voir en général DiLLERY (2005).

44 PritchetT (1979), p. 54-56; Greenwalt (1982), p. 24-25; PARKer (2000). Cf. aussi le témoignage de Frontin, Strat. I, 11, 14.

45 Xén., Anab. V, 6, 29. Voir aussi Bruit Zaidman (2001), p. 67-71; Burkert (2005), p. 7; PARKER (2004) ainsi que la contribution de L. Bruit dans le présent dossier.

46 Voir Arrien, Anab. VII, 18, 2, où il prédit la mort de Perdiccas et d'Antigone; cf. Appien, BC II, 152.

47 Berve (1926), no 618; HeCKel (2006), p. 194.

48 Voir PASCHIDIS (2006). Sur cette dualité au niveau des récits mythologiques, voir MALLIOS (2011), p. 31, qui ajoute également une troisième composante, celle des indigènes du pays 
image complète de la religion macédonienne, il pourrait être utile de mener une réflexion de type prosopographique sur la pratique divinatoire dans cette région.

Originaire d'Amphipolis, Pythagore naquit probablement peu avant ou peu après la conquête de la ville par Philippe II en 357 av. J.-C.49. Le frère de Pythagore, Apollodore, était compagnon d'Alexandre, ce qui laisse entendre que sa famille devait être assez puissante. Lui-même faisait partie du cercle des devins qui gravitaient autour du roi, et dont le plus important était Aristandre de Telmessos, en Carie $^{50}$. Cependant, il est fort probable que cette position incluait également des devoirs militaires, étant donné que les devins pouvaient aussi être des experts en ce domaine ${ }^{51}$. On sait ainsi qu'Alexandre, à la veille de grandes batailles, discutait des détails des opérations avec les devins qui étaient à son service, et surtout avec Aristandre ${ }^{52}$. Il existerait même des représentations iconographiques qui soulignent l'importance de ce dernier personnage dans le domaine militaire : si l'interprétation de Paolo Moreno est juste, alors la personne qui se trouve à côté d'Alexandre et porte un casque avec une couronne dorée, sur la fameuse mosaïque d'Alexandre, serait justement Aristandre de Telmessos ${ }^{53}$.

Nous ne savons pas si Pythagore fit partie du groupe des devins au service d'Alexandre dès le début de l'expédition asiatique, mais c'est une possibilité étant donné que le point de départ en fut précisément Amphipolis, lieu où l'armée et la flotte firent leur jonction pour partir vers l'est ${ }^{54}$. De plus, Alexandre avait l'intention d'y faire ériger après la fin de l'expédition un grand temple en l'honneur d'Artémis Tauropole ${ }^{55}$. Ces détails nous renvoient immanquablement au moment du départ d'une autre expédition "panhellénique», celle qui partit d'Aulis pour Troie : la même divinité y joua un rôle important. Il est connu qu'Alexandre se voulait un nouvel Achille, et les similitudes que nous avons relevées sont peutêtre l'indice d'une réminiscence homérique. Peut-être Pythagore faisait-il partie des officiers en charge des préparatifs, d'autant que le rôle des devins lors du départ de l'expédition est bien attesté56. Du reste, pour continuer dans la voie des

macédonien, c'est-à-dire les populations qui y habitaient avant la colonisation grecque et l'expansion du royaume; à ce sujet, voir aussi TiVERIOS (2008).

49 Sur l'importance de cette conquête et l'interaction entre «monarchic and local elements » au niveau administratif et religieux voir ERRINGTON (2007) et MARI (2007). Sur l'histoire d'Amphipolis jusqu’à la conquête macédonienne voir aussi LORBER (1990), p. 1-7.

${ }^{50}$ Sur Aristandre, voir KING (2004) et NICE (2005).

51 PritchetT (1979), p. 56-57; Dillery (2005), p. 200-209; NiCE (2005), p. 89, n. 15; BOWdeN (2005), p. 148-150; Flower (2008), p. 153-187; Flower (2008 a), p. 202. Pourtant, dans les récits des historiens d'Alexandre, nous n'avons pas d'informations précises sur une intervention dans les affaires militaires des devins en tant que soldats ou stratèges.

\footnotetext{
52 Plut., Alex., 31, 9; Quint. Curt., IV, 13, 15.

53 Moreno (2001), p. 19 et p. 51, table V.

${ }^{54}$ Arrien, Anab. II, 3.

${ }^{55}$ Diod., XVIII, 4. Voir aussi LORBER (1990), p. 13.

56 Arrien, Anab. I, 11.
} 
hypothèses, le devin d'Amphipolis ne pouvait pas ne pas connaître les traditions sur le culte dionysiaque et orphique lié au mont Pangée ${ }^{57}$.

Voyons maintenant quelques détails qui concernent la pratique même de la divination par Pythagore. Il était normal que l'examen des entrailles se fasse en présence des personnes concernées et en toute connaissance de cause de leur part. Or, ce n'est pas le cas dans l'épisode de Babylone, et cela nous amène à nous demander si la présence et la conscience de l'intéressé sont requises dans le rituel de la manteia ${ }^{58}$. Les rites décrits par Plutarque semblent renvoyer plutôt aux procédés des mages babyloniens. Si tel est bien le cas, on pourrait parler d'une influence exercée par les prêtres babyloniens sur les experts religieux grecs. Il se peut que les devins grecs, en rivalité avec leurs collègues babyloniens à la cour d'Alexandre, se soient adaptés aux circonstances nouvelles, afin de pouvoir maintenir leur place privilégiée ${ }^{59}$. Il semble que Pythagore y a plutôt réussi, puisqu'il a su s'imposer comme instance divinatoire, surtout en matière de pronostics mortuaires, auprès d'une série de Diadoques pendant toute une génération après la mort d'Alexandre.

Cette interprétation ne prend cependant pas en compte le fait que les mages, bien avant Alexandre, étaient entrés dans le monde des cités et des royaumes grecs, et que la Macédoine avait subi dès avant l'expédition asiatique l'influence achéménide $^{60}$. En tout cas, l'image qui ressort de cet épisode est celle d'un Pythagore en robe de mage, pour paraphraser M. Carastro ${ }^{61}$, et voilà peut-être ce que Plutarque voulait suggérer à ses lecteurs en préférant pour son nom la graphie Pythagoras avec un $y$ et non pas, comme chez Arrien, la graphie Peithagoras. Il convient ici de faire la comparaison avec le peritissimus vatum, Aristandre, le plus célèbre des devins d'Alexandre. Il semble que la figure d'Aristandre ait été également réinterprétée pendant les $\mathrm{I}^{\mathrm{er}}$ et $\mathrm{II}^{\mathrm{e}}$ siècles ap. J.-C. Les testimonia sur la vie et l'œuvre de ce devin ${ }^{62}$ soulignent l'aspect néoplatonicien d'Aristandre, caractérisé par un savoir ésotérique et philosophique.

Quoi qu'il en soit, sous l'une ou l'autre forme, le nom de Pythagoras était un omen: la graphie avec un $y$ insiste sur le caractère charismatique du devin, celle avec $e i$ souligne la capacité de persuader son audience, le cas échéant les soldats ou les stratèges ${ }^{63}$.

57 Voir GARTZIOU-TATTI (1999).

58 Selon Quinte Curce, l'absence du roi pendant ce rituel était conforme aux lois ancestrales : VII, 7, 8: mos erat haruspicibus exta sine rege spectare et, quae porterendur, referre; sur ce point voir plus bas. Voir aussi les remarques de Flower (2008), p. 130-131.

${ }^{59}$ FredRiCKSMEYR (2003), p. 269.

${ }^{60}$ Sur la Macédoine sous les Achéménides, voir SPAwFORTH (2007), p. 92. Voir aussi BuRKERT (2004), p. 117-118, au sujet du papyrus de Derveni.

${ }^{61}$ Carastro (2006), p. 209 (au sujet de Socrate). Cf. Cic., De div. I, 5.

62 Voir NiCE (2005), p. 95-97.

${ }^{63}$ Sur l'importance du nom pour un devin, voir Flower (2008), p. 47. 


\section{Réalité et représentation : un regard romain sur la manteia macédonienne?}

Ces considérations nous conduisent à aborder la question des représentations et topoi littéraires, dont en réalité la divination par le foie fait partie intégrante. Dans les textes présentés, nous avons affaire à des témoignages d'auteurs qui réfléchissent sur la religiosité (Plutarque), qui s'inspirent d'auteurs classiques (Xénophon, qui constitue une référence pour Arrien, insiste sur le rôle de la divination) et qui écrivent alors que la monarchie macédonienne n’était plus qu'un souvenir assez lointain. Il convient encore de leur ajouter Quinte-Curce, qui se montre très critique envers la divination en général ${ }^{64}$.

Pour tous ces auteurs historiens d'Alexandre, l'empereur romain constituait le paradigme du comportement monarchique, et il est connu que la réalité romaine influença la rédaction de la Vie d'Alexandre et de l'Anabase ${ }^{65}$. Cela s'avère plus clair chez Quinte-Curce ${ }^{66}$, qui insiste sur la différence entre affaires publiques et affaires privées, différence qui ne devait pas être aussi nette sous les Argéades ${ }^{67}$. Mais dans l'Empire romain, les lois tendirent de plus en plus à considérer comme crimes publics des actes qui paraissaient relever d'un caractère privé68. C'est pourquoi il faut être très prudent en lisant Quinte Curce, lorsqu'il évoque « la loi ancestrale » et l'ordre donné par Alexandre de faire un sacrifice privé69.

Pour toutes ces raisons, je pense comme M. Flower que le type de divination décrite plus haut par Plutarque, avec les conséquences politiques et juridiques qu'elle entraînait (la dénonciation contre Apollodore), renvoie plutôt à la divination pro salute principis ${ }^{70}$, alors qu'il est notoire que toute divination au sujet de l'empereur était devenue une pratique strictement contrôlée et interdite aux particuliers $^{71}$. Il faudrait par conséquent être conscient de cet écart entre la pratique de la divination dans la monarchie macédonienne et sa représentation dans les sources littéraires ${ }^{72}$.

${ }^{64}$ Quint. Curt., VII, 7, 8 : bumanarum mentium ludibria.

65 Sur la « romanisation » d'Alexandre à travers la romanisation d'Aristandre, voir NICE (2005), p. 97, qui examine les pratiques sacrificielles évoquées par Quinte Curce et le vocabulaire qu'il utilise. Ces deux éléments trahissent une influence romaine et étrusque. Voir aussi KOULAKIOTIS (2010).

66 Voir BAYNHAM (2001), p. 114-115.

${ }^{67}$ Quint. Curt., VII, 23 : Non rex, inquit sed privatus [sum] sacrificium ut faceres, mandavi. Cf. aussi Arrien, Anab. VII, 7, 9.

68 Voir GrodzynSKI (1974); DESANTI (1988) et HAACK (2002), et en général NORTH (1990).

${ }^{69}$ Quint. Curt., VII, 7, 8 et VII, 7, 23-27. Voir aussi n. 58.

${ }^{70}$ Voir Paulus, Sent. V, 21, 3; cf. Tac., Ann. II, 27, 2; 30, 1-2; 32, 3.

71 GRODZYNSKI (1974), p. 268.

72 Voir les remarques de FLOWER (2008), p. 21 sur la distinction entre « literary religion and practiced religion ». 
En guise de conclusion, je dirais que l'importance de la divination, des personnes et des institutions qui y sont impliquées, ainsi que de leur rôle dans la réalité et l'idéologie de la royauté macédonienne me semble donnée, mais que ce terrain reste encore largement à explorer ${ }^{73}$. Ici, à partir du mythe fondateur de la dynastie et d'un épisode, à mon avis significatif, de l'expédition d'Alexandre, je n'ai pu réaliser qu'une esquisse nécessairement incomplète. Néanmoins, celle-ci dessine l'image d'une monarchie inscrite dans la tradition de la royauté hérö̈que, enrichie au contact des pratiques des cités, mais aussi de celles des empires perse et ultérieurement romain.

Elias KOULAKIOTIS

Université d'Ioannina,

Faculté d'Histoire et Archéologie,

GR - 45510 IOANNINA

Courriel:koulakio@cc.uoi.gr

\section{Bibliographie}

Abramenko, A., «Der Fremde auf dem Thron. Die letzte Verschwörung gegen Alexander den Großen », Klio 82 (2000), p. 361-378.

Auberger, J., Historiens d'Alexandre, Paris, 2001.

Aubriot, D., "Quelques observations sur la religion d'Alexandre (par rapport à la tradition classique) à partir de Plutarque et d'Arrien », Mètis n.s. 1 (2003), p. 225-249.

BAEGE, W., De Macedonum Sacris, Halle, 1913.

Baynham, E., Alexander the Great. The Unique History of Quintus Curtius, Ann Arbor, 2001.

Berve, H., Das Alexanderreich auf prosopographischer Grundlage II : Prosopographie, Munich, 1926.

Bonnechere, P., «Divination », in D. Ogden (éd.), A Companion to Greek Religion, London, 2010, p. 145-159.

Bosworth, A.B., A Historical Commentary on Arrian's History of Alexander, vol. I, Oxford, 1980.

—, Conquest and Empire. The Reign of Alexander the Great, Cambridge, 1988.

Bowden, H., Classical Athens and the Delphic Oracle. Divination and Democracy, Cambridge, 2005.

BRANDENSteIN, W., «Die Reichsgründersage des makedonischen Herrscherhauses», in B. SuTTER (éd.), Festschrift J.F. Schütr, Graz \& Cologne, 1954, p. 54-58.

Briant, P., Histoire de l'empire perse de Cyrus à Alexandre, Paris, 1996.

BRIANT, P., "Alexandre à Babylone: images grecques, images babyloniennes", in L. HARFLANCNER, C. KAPPLER, F. SUARD (éd.), Alexandre le Grand dans les traditions médiévales occidentales et proche-orientales, Paris, 1999, p. 23-32.

Bruit Zaidman, L., Le commerce des dieux. Eusebeia, essai sur la piété en Grèce ancienne, Paris, 2001.

Buraselis, K., ANEZIRI, S., « Die griechische und hellenistische Apotheose «, ThesCRA II (2005), p. $158-185$.

BurkerT, W., Babylon, Memphis, Persepolis. Eastern Contexts of Greek Culture, Harvard, 2004.

—, «Mantik in Griechenland », ThesCRA III (2005), p. 1-16.

Carastro, M., La cité des mages, Paris, 2006.

${ }^{73}$ L'apport de l'archéologie y sera sans doute précieux. Pour leur aide dans l'étude présente j’aimerais remercier A. Zografou, A. Helmis, G. Mallios et P. Kossmann. 
CArLIER, P., "Homeric and Macedonian Kingship », in R. Brock, S Hodkinson (éd.), Alternatives to Athens. Varieties of Political Organization and Community in Ancient Greece, Oxford, 2000, p. 259-268.

Carney, E., Olympias, Mother of Alexander the Great, New York/London, 2006.

—, "Arrian's sources and reliability ", in J. Romm (éd.), The Landmark Arrian. The Campaigns of Alexander, New York, 2010, p. 325-332.

Chevutschi, L., Les légendes dynastiques de Grèce du Nord (Macédoine et Epire), thèse de doctorat, Université Paris X, Paris, 1991.

Chirassi Colombo, I., «Teras ou les modalités du prodige dans le discours divinatoire grec: une perspective comparatiste», in S. GEORGOUd, R. KOCH PieTtre, F. SCHMidT (éd.), La raison des signes: présages, rites, destin dans les sociétés de la Méditerranée ancienne, Leiden, 2012, p. 221-253.

Collard, C. Cropp, M, Gibert, J., Euripides. Selected Fragmentary Plays, vol. II, Oxford, 2005.

DeSANTI, L., «La repressione della scienza divinatoria nell'età del principato », in A. BURDESE, (éd.), Idee vecchie e nuove sul diritto criminale romano, Padova, 1988, p. 225-240.

Descamps-Lequime, S. (dir.), Au royaume d'Alexandre le Grand. La Macédoine antique, Paris, 2011.

Detienne, M., Les maîtres de vérité dans la Grèce archaique, Paris, 1973.

Dillery, J., "Chresmologues and Manteis: Independent Diviners and the Problem of Authority ", in S.I. Johnston, P. Struck (éd.), Mantike. Studies in Ancient Divination, Leiden, 2005, p. 167-231.

Drougou, S., "Aigai », in S. Descamps-Lequime (dir.), Au royaume d'Alexandre le Grand. La Macédoine antique, Paris, 2011, p. 658.

Engels, J., « Macedonians and Greeks », in J. Roisman, I. Worthington (éd.), A Companion to Ancient Macedonia, London, 2010, p. 81-98.

ERrington, M., «The Importance of the Capture of Amphipolis for the Development of the Macedonian City ", Ancient Macedonia 7 (2007), p. 275-272.

Flower, M., "Not Great Man History: Reconceptualizing a Course on Alexander the Great », $C W 100.4$ (2007), p. 417-423.

—, The Seer in Ancient Greece, Berkeley, 2008.

-, «The Iamidae: A Mantic Familly and Its Public Image», in B. Dignas, K. Trampedach (éd.), Practitioners of the Divine. Greek Priests and Religious Officials from Homer to Heliodorus, Washington, 2008a, p. 187-206.

Fontenrose, J., The Delphic Oracle. Its Responses and Operations with a Catalogue of Responses, Berkeley, 1978.

FredricksmeYer, E.A., «The Ancestral Rites of Alexander the Great », CPh 61 (1968), p. 179182.

-, «Alexander's religion and divinity ", in J. Roisman (éd.), Brill's Companion to Alexander the Great, Leiden, 2003, p. 253-278.

Freyburger, G., Scheid, J., Cicéron. De la divination, Paris, 1992.

GalanAKis, Y., "Aegae : 160 years of archaeological research », in Heracles to Alexander the Great, 2011, p. 49-58.

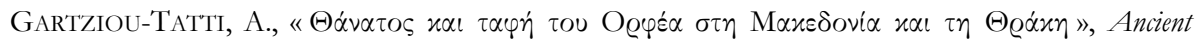
Macedonia VI (1999), p. 439-451.

Georgoudi, S., Préface à Auguste Bouché Leclerq, Histoire de la divination dans l'Antiquité, Grenoble, 2003, p. 5-17.

—, «Des sons, des signes et des paroles: la divination dans l'oracle de Dodone», in S. Georgoudi, R. Koch PietTre, F. SChmidt (éd.), La raison des signes : présages, rites, destin dans les societés de la Méditerranée ancienne, Leiden, 2012, p. 55-90.

Greenwalt, W., « A Macedonian Mantis », AncW 5 (1982), p. 17-25.

—, «The Introduction of Caranus into the Argead King List », GRBS 26 (1985), p. 43-49. 
—, «Herodotus and the Foundation of Argead Macedonia », AncW 13 (1986), p. 123-128.

—, «A Solar Dionysus and Argead Legitimacy », AncW 25 (1994), p. 3-8.

Grodzynski, D., « Par la bouche de l'empereur. Rome, IVe siècle », in Vernant (1974), p. 267-294.

HAAck, M.-L., «Haruspices publics et privés. Tentative d'une distinction », REA 104 (2002), p. $111-133$.

Hamilton, J.R., Plutarch, Alexander, Second Edition, Foreword and Bibliography by Philip A. Stadter, London, 1999.

Harder, M.A., "Euripides" Temenos and Temenidai », in H. Hofmann, Fragmenta Dramatica. Beiträge zur Interpretation der griechischen Tragikerfragmente und ibrer Wirkungsgescbicbte, Göttingen, 1991, p. 117-135.

HARrISON, Th., Divinity and History, Oxford, 2001.

Harvey, F.D., « Herodotus 1.78 and 84: Which Telmessos? «, Kernos 4 (1991), p. 245-258.

Hatzopoulos, M., Macedonian Institutions under the Kings. A Historical and Epigraphical Study, vol. III, Athènes, 1996.

-, «Herodotus (8.137-8), the Manumissions from Leukopetra, and the Topography of the Middle Haliakmon Valley », in P. Derow, R. PArker (éd.), Herodotus and His World. Essays From a Conference in Memory of George Forrest, Oxford, 2003, p. 203-218.

—, La Macédoine : Géographie Historique, Langue, Cultes et Croyances, Institutions, Paris, 2006.

HeCKEL, W., Who's Who in the Age of Alexander the Great, London, 2006.

Heracles to Alexander the Great. Treasures from the Royal Capital of Macedon, a Hellenic Kingdom in the Age of Democracy, catalogue d'exposition, Ashmolean Museum, Oxford, 2011.

KAPETANOPOULOS, E. 2010. «Karanos-Perdikkas-Arkhelaos », http://www.history.ccsu.edu/ elias/karanosperdikkas.pdf (23 mars 2012)

Kazazis, J.N., Herodotos' Stories and History: A Proppian Analysis of his Narrative Technique, Unpublished Doctoral Thesis, University of Illinois at Urbana Campain, 1978.

KeTt, H., Prosopographie der historischen griechischen Manteis bis auf die Zeit Alexanders des Grossen, Diss. Erlangen, 1966.

KING, C., Alexander and Divination: Dreams, Omens, and Aristander of Telmessus in the Alexander Historians, thèse de doctorat, Providence, 2004.

—, «Macedonian kingship and other political institutions », in J. ROISMAN, I. WORTHINGTON (éd.), A Companion to Ancient Macedonia, London, 2010, p. 373-391.

Kleinknecht, H., « Herodot und die Makedonische Urgeschichte », Hermes 94 (1966), p. 134146.

Koulakiotis, E., Genese und Metamorphosen des Alexandermythos im Spiegel der griechischen nichthistoriographischen Überlieferung bis zum 3. Jh. n. Chr., Konstanz, 2006.

-, «Approaching cultural Identities in the Roman World: Alexander the Great and the other Macedonian Kings in the literary sources », in I. STEFAnidis, V. Vlasidis, E. KofOS (éd.), Macedonian Identities Through Time, Thessalonique, 2010 p. 63-92.

KotTARidi, A., "The Legend of Macedon: a Hellenic Kingdom in the age of democracy «, in Heracles to Alexander the Great, 2011, p. 1-24.

-, 2011a. «Queens, princesses and high priestesses: the role of women at the Macedonian courts, in Heracles to Alexander the Great, p. 93-126.

—, 2011b. «Aegae : The Macedonian metropolis », in Heracles to Alexander the Great, p. 153-166.

Kunrt, A., The Persian Empire, London/New York, 2007.

Le Bohec-Bounet, S., «Réflexions sur la place des femmes dans la Macédoine antique », in A.-M. Guimier-Sorbets, M.B. Hatzopoulos, Y. Morizot (éd.), Rois, cités, nécropoles. Institutions, rites et monuments en Macédoine, Athènes, 2006, p. 187-198.

LiamPI, K., Der makedonische Schild, Bonn, 1998. 
LORBER, C., Amphipolis. The civic coinage in silver and gold, Los Angeles, 1990.

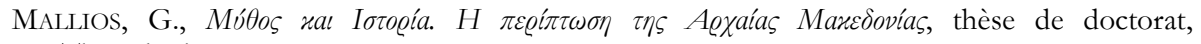
Thessalonique, 2011.

MARI, M., Al di là dell' Olimpo. Macedoni e grandi santuari della Grecia dall' età arcaica al primo ellenismo, Athènes, 2004.

-, "The ruler cult in Amphipolis and the Strymon valley », in A. IACOvidou (éd.), Thrace in the Graeco-Roman World, Athènes, 2007, p. 371-386.

Moreno, P., Apelle, Milano, 2001.

Müller, S., «Inventing Traditions. Genealogie und Legitimation in den hellenistischen Reichen », in H. BrandT, K. KÖHLER, U. SIEWERT (éd.), Genealogisches Bewusstsein als Legitimation, Bamberg, 2009, p. 61-82.

NiCE, A., «The Reputation of the 'Mantis' Aristander », Acta Classica 48 (2005), p. 87-102.

North, J., "Diviners and Divination at Rome», in M. BEARD, J. NorTH (éd.), Pagan Priests: Religion and Power in the Ancient World, London, 1990, p. 49-71.

Ogden, D., Alexander the Great. Myth, Genesis and Sexuality, Exeter, 2011.

PABST, S., «Bevölkerungsbewegungen auf der balkanhalbinsel am Beginn der früheisenzeit und die Frage der Ethnogenese der Makedonen », JdI 124 (2009), p. 1-73.

Parke, H.W., Wormell, D.E.W., The Delphic Oracle. I. The History II. The Oracular Responses, Oxford, 1956.

PARKER, R., «Sacrifice and the Battle », in H. VAN WEEs (ed.), W ar and Violence in Ancient Greece, London, 2000, p. 299-314.

-, «One man's piety: the religious dimension of the Anabasis », in R. LANE Fox (éd.), The Long March, New Haven, 2004, p. 131-153.

PASCHIDIS, P., «The interpenetration of civic elites and court elite in Macedonia ", in A.-M. Guimier-Sorbets, M.B. Hatzopoulos, Y. Morizot (éd.), Rois, cités, nécropoles. Institutions, rites et monuments en Macédoine, Athènes, 2006, p. 251-267.

PEARSON, L., The Lost Histories of Alexander the Great, Chicago, 1960.

PEDECH, P., Historiens compagnons d'Alexandre, Paris, 1984.

Pelling, Ch., Plutarch and History: Eighteen Studies, Swansea, 2002.

PRITCHETT, W.K., The Greek State at War, vol. III, Berkeley, 1979.

Reynolds, A.C., Perdiccas I to Alexander I: The First Ten Temenid Kings of Argead Macedonia, thèse de doctorat, Edmonton, 1990.

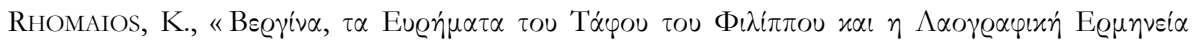

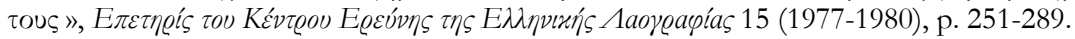

RiTTER, H.W., «Zur Sternsymbolik im antigonidischen und argeadischen Makedonien », Archaiognosia 2 (1981), p. 159-205.

Roth, P.A., Mantis. The Nature, Function, and Status of a Greek Prophetic Type, Diss. Bryn Mawr College, 1982.

SMELIK, K., «Omina mortis », Talanta 10/11 (1978/79), p. 92-111.

Sourvinou-Inwood, C., «Greek Perceptions of Ethnicity and the Ethnicity of the Macedonians ", in L. Moscati Castelnuovo (éd.), Identità e prassi storica nel Mediterraneo Greco, Milano, 2002, p. 173-203.

-, Hylas, the Nymphs, Dionysos and Others. Myth, Ritual, Ethnicity, Uppsala, 2005.

SPAWFORTH, A., "The Court of Alexander the Great between Europe and Asia », in A. SPAWFORTH (éd.), The Court and Court Society in Ancient Monarchies, Cambridge, 2007, p. 82-121.

Sprawski, S., «The Early Temenid Kings to Alexander I », in J. ROISMAN, I. WORTHINGTON (éd.), A Companion to Ancient Macedonia, London, 2010, p. 127-144. 
ThemL, N., «Temenidas Herodotiano. Mito E Rito De Fundação da realeza dos macedônios », Hélade 1 (2000), p. 53- 64.

Tiverios, M., « Greek Colonisation of the Northern Aegean », in G. TSETSKHLADZE (éd.), Greek Colonisation vol. 2, Leiden/Boston, 2008, p. 1-54.

Tripodi, B., «L“"emblema” della casa reale macedone », in Ancient Macedonia IV (1986), p. 653-60.

—, "Tipologia e ideologia di Perdicca, primo 'Fondatore' della regalità macedone ", in Ancient Macedonia V, vol. 3 (1993), p. 1623-1630.

Vernant, J.-P. (dir.), Divination et rationalité, Paris, 1974.

WirTh, G., « Anmerkungen zur Arrianbiographie », Historia 13 (1964), p. 209-245.

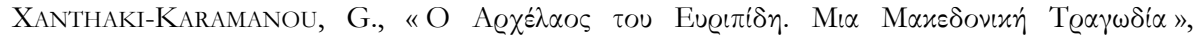
Паৎvaббós 35 (1993), p. 510-533.

Zambrini, A., "The Historians of Alexander the Great », in J. Marincola (éd.), A Companion to Greek, and Roman Historiography, vol. I, London, 2007, p. 210-220.

ZAHRnt, M., «Die Entwicklung des makedonischen Reiches bis zu den Perserkriegen », Chiron 14 (1984), p. 325-368. 